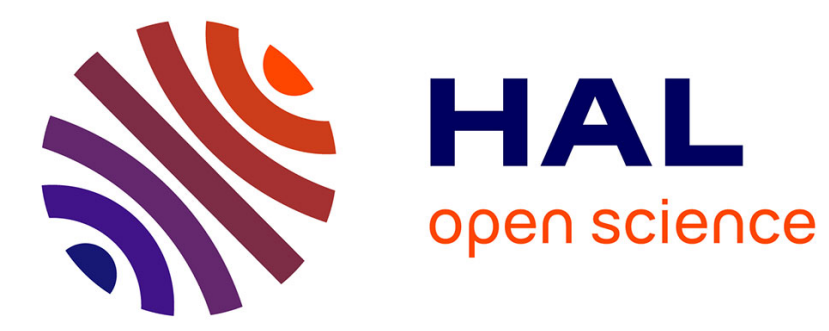

\title{
Do rotating dust stars exist in general relativity?
}

Herbert Pfister

\section{To cite this version:}

Herbert Pfister. Do rotating dust stars exist in general relativity?. Classical and Quantum Gravity, 2010, 27 (10), pp.105016. 10.1088/0264-9381/27/10/105016 . hal-00594829

\section{HAL Id: hal-00594829 \\ https://hal.science/hal-00594829}

Submitted on 21 May 2011

HAL is a multi-disciplinary open access archive for the deposit and dissemination of scientific research documents, whether they are published or not. The documents may come from teaching and research institutions in France or abroad, or from public or private research centers.
L'archive ouverte pluridisciplinaire HAL, est destinée au dépôt et à la diffusion de documents scientifiques de niveau recherche, publiés ou non, émanant des établissements d'enseignement et de recherche français ou étrangers, des laboratoires publics ou privés. 


\title{
DO ROTATING DUST STARS EXIST IN GENERAL RELATIVITY?
}

\author{
Herbert Pfister \\ Institut für Theoretische Physik, Universität Tübingen, \\ Auf der Morgenstelle 14, D 72076 Tübingen, Germany \\ E-mail: herbert.pfister@uni-tuebingen.de
}

\begin{abstract}
If rotating dust stars would exist in general relativity, they would represent examples of an improbable complete balance between the attractive quasi-Newtonian force (gravitoelectricity) and the repulsive gravitomagnetism. However, nonexistence proofs are available hitherto only for some dust "stars" extending to infinity, and for isolated dust stars of a very restricted class. By analyzing the lines of constant generalized Newtonian potential $U$ in the interior and exterior of a large class of (hypothetical) stationary and axisymmetrically rotating dust stars in general relativity, we find that the existence of such stars can be disproved as soon as minima of the potential $U$ in the exterior vacuum region can be excluded. We present some ideas how this minimum-problem could be attacked, and we summarize the present knowledge about Newtonian and Einsteinian rotating dust systems.
\end{abstract}

PACS numbers: 04.20.Cv, 04.20.Ex, 04.40.Dg

\section{Introduction}

There are at least three reasons why the question of existence of rotating dust stars in general relativity is particularly delicate and mathematically difficult.

a) It has to be clarified why such stars presumably cannot exist, whereas already a small pressure, respectively a small pressure gradient at the surface can stabilize a star. We argue that a decisive difference between the two cases (a tiny cause with drastic consequences!) shows up in the level lines of the generalized Newtonian potential near the rotation axis (in a metric form which provides maximal similarity with the Newtonian case): Whereas for stars with pressure these lines are everywhere orthogonal to the rotation axis, for dust stars they are parallel to the rotation 
axis in the matter region near the axis, what leads to a type of singularity of the level lines at the "poles" where the axis leaves the matter region. (Another difference between the two cases is of course that for stars with pressure the surface is fixed as the location with pressure $p=0$, whereas for dust stars the surface geometry would remain largely arbitrary.)

b) The quasilocal difference in the structure of these level lines alone can, however, not explain why rotating dust stars should not exist: In Newtonian gravity, where the level lines of the potential for (hypothetical) dust stars have the same structure as described above, it was shown by an explicit example [1] that a rotating dust star can be stabilized by exterior (strained) matter which can be arbitrarily far off the dust region. Although it will be difficult to construct such an explicit example in general relativity, it is clear that also in general relativity the problem of existence of a rotating dust star is a genuinely global one. We find that the existence of rotating dust stars can be disproved as soon as minima of the generalized Newtonian potential $U$ in the exterior vacuum region can be excluded, a question which seems to be open, notwithstanding a huge literature and considerable progress on the stationary and axisymmetric vacuum Einstein equations (Ernst equation, integrable systems, algebro-geometric methods). In Sec. 4 we present some ideas how the question of $U$-minima can be attacked. And although we are not yet successful in this goal, our article may motivate other researchers to look into this mathematical problem.

c) It has been known since long time, and by quite different proof methods, that isolated Newtonian rotating dust stars cannot exist. (See below.) But there is no chance to simply generalize these proofs into the regime of general relativity. On one hand we know that general relativity comprises physical phenomena completely absent from Newtonian gravity: gravitational collapse, gravitational waves, and, particularly interesting for our stationary problem, gravitomagnetism. It has been argued already long ago [2] that the gravitomagnetic potential can have a repulsive or pressure-like effect for rotating stars, and we will partly strengthen these arguments. However, we suspect that the effect of this gravitomagnetic potential can never be strong enough to stabilize a rotating dust star. (In the weak field limit, and for rotating shell-like and ring-like objects, it was shown in [3] that a complete compensation between gravitational attraction and gravitomagnetic repulsion is possible only for unrealistic limiting cases.) On the other hand, the mathematical structures of Newtonian gravity and general relativity are very different. And, although considerable progress has been made here, mainly through the work of the late Jürgen Ehlers [4], there are still many open problems concerning the Newtonian limit of solutions of general relativity, and even more so concerning generalizations of existence- or non-existence-results from Newtonian gravity to general relativity. 
Before we present (in Sec. 2) in detail the mathematical structure of the problem of rotating dust stars in general relativity, and derive (in Sec. 3) consequences for the level lines of the generalized Newtonian potential as a possible first step for a nonexistence proof for such objects, we like to summarize here what is already known about rotating dust systems in Newtonian and Einsteinian gravity.

In Newtonian gravity there exist convincing and quite different (physical, geometrical, and analytic) arguments why stationary and axisymmetrically rotating dust stars cannot exist. The possibly simplest argument (see, e.g., [5]) uses a plane orthogonal to the rotation axis and "shifts" it from infinity until it touches the dust matter at one or several points $P$. At $P$ the gravitational attraction points into the half space containing the dust matter, whereas the centrifugal force is tangent to the plane. Therefore the dust particle at $P$ cannot be stationary. Obviously this argument breaks down if the matter covers only a two-dimensional disk orthogonal to the axis. And indeed, rotating dust disks do exist in Newtonian [6] as well as in Einsteinian gravity [7]. In this article we confine ourselves to (hypothetical) dust stars with spherical topology, and do not address possible star solutions with toroidal or more complicated topology. To our knowledge the first analytic proof for the nonexistence of axisymmetrically rotating dust stars in Newtonian theory was given by Bonnor [2]: The gravitational potential $U$ is independent of the axiscoordinate $z$ in the dust region, is continuously differentiable across the surface, and analytic in the exterior. Therefore $U_{, z}$ fulfills the Laplace equation in the exterior, is zero at infinity and at the star surface, and therefore is identically zero in the exterior. Together with $U \longrightarrow 0$ at infinity, this leads to $U \equiv 0$ in the exterior, and, e.g., through the Poisson integral, to energy-density $\epsilon \equiv 0$. In [1], Sec. 2.4, we have given an alternative analytic nonexistence proof, using a minimum principle, which has a better chance to show the way to an extension to general relativity, e.g., through the consideration of $U$ level lines.

In general relativity the first dust solution (dust particles in stationary, cylindrically symmetric, and rigid rotation about a symmetry axis) was discovered by Lanczos [8], and discussed as a cosmological solution. The solution was rediscovered and generalized by van Stockum [9]. Winicour [10] succeeded in showing that even for differential rotation the Einstein equations in the dust reduce to quadratures, and that the general solution depends upon an arbitrary axisymmetric solution of the flat Laplace equation together with an arbitrary function of one variable. Bonnor [2] hinted to the fact that the gravitomagnetic potential of general relativity leads for rotating dust systems to a repulsive "force" in the axis direction, counteracting the Newtonian gravitational attraction. However, in Bonnor's explicit example of a rigidly rotating dust cloud extending tenuously to infinity (hereby realizing asymptotic flatness), the stabilization results not so much from this grav- 
itomagnetic potential but from a singularity of the solution at the center. (See [1], footnote 12.) Indeed, Caporali [5] could show, using the results of Winicour [10], that even for differential axisymmetric rotation of dust no nontrivial, singularityfree, and asymptotically flat solution exists as long as the energy-density is non-zero in an open neighborhood of the whole axis. In detail he proves that the total mass $M$ of such a system has to be zero, and therefore $\epsilon \equiv 0$, as long as we do not admit negative energy densities. Frauendiener [11] could show that stationary and axisymmetric dust solutions (and more general solutions with non-negative two-trace of the energy-momentum tensor) do not exist on spatially compact manifolds. Recently, Gürlebeck [12] has, for the first time, presented some nonexistence results for rotating dust, occupying only a finite space part $\Omega$ around the axis, and he does this - maybe surprisingly — by analyzing the Einstein equations only in the dust region. In detail, he considers dust in stationary, axisymmetric and rigid rotation. Technically he works in the corotating coordinate system (as already van Stockum [9] did), and transforms the gravitomagnetic potential — similar to the formalism of the Ernst equation in vacuum - to a new potential fulfilling the Laplace equation. Hereby he can show that the solution in $\Omega$ is already determined by a largely arbitrary energy density $\epsilon$ on the axis. (This is, due to the action of gravitomagnetism, in contrast to the Newtonian case where rigid rotation implies constant $\epsilon$.) Gürlebeck then shows that there do not exist solutions with $\epsilon=0$ on $\partial \Omega$, or with $\epsilon=$ const. in $\Omega$. It should, however, be clear that these methods do not work for dust stars in differential rotation. And also for rigid rotation, and $\epsilon>0$ on $\partial \Omega$, or $\epsilon \neq$ const. in $\Omega$, it will be necessary to consider the global problem with an appropriate exterior vacuum solution.

We consider in the following (in Secs. 2 - 4) rotating dust stars in general relativity which can have differential rotation with an angular velocity of arbitrary analytic dependence on the coordinates. We confine ourselves to star shapes which satisfy a sphere condition at least at the north pole, i.e., which do not have a cone or cusp there. Furthermore, we assume that the energy density of the dust at the north pole is either positive, or has one nontrivial derivative in the axis direction.

At the end of this Introduction we should like to add a remark on stationary and axisymmetrically rotating stars with pressure: Although such systems seem to make up (in good approximation) most of the visible matter in our universe, the theoretical state of art for these systems is still in bad shape in Newtonian and Einsteinian gravity. Only for static stars, and for slowly rotating stars with constant density or with polytropic equation of state in Newtonian theory, existence proofs and some results about their physical properties are available. In general relativity, the only existence proof for rotating stars was given by Heilig [13]. However, 
since this work is based on the implicit function theorem, it is hard to judge how relativistic or compact, and how fastly rotating these stars can be. And no real progress on these questions has been made since the publication of [13]. In [14] we have presented mathematical techniques which should be applicable to a wide class of rotating stars, but details are still waiting for their elaboration.

\section{Setup of the mathematical problem}

We confine ourselves to stars consisting of ideal fluid (with or without pressure $p$ ) in stationary and axisymmetric motion along the orbits of the axial Killing vector $\partial_{\varphi}$, i.e., we exclude any convective motion in the direction of the other spatial coordinates $\rho, z$. We allow, however, for differential rotation, i.e., the angular velocity $\omega$ of the fluid particles may depend on $\rho, z$. With the fluid four-vector $u^{\mu}=u^{0}(1,0,0, \omega)$, and with the energy density $\epsilon$, the energy-momentum tensor has the structure

$$
T^{\mu \nu}=(\epsilon+p) u^{\mu} u^{\nu}+p g^{\mu \nu} .
$$

It is known since a long time [15] that for such systems the metric attains a "block structure" with separate parts in the $(\rho, z)$ - and $(t, \varphi)$ - spaces. One popular form is the Lewis-Papapetrou metric (see, e.g., [16])

$$
d s^{2}=g_{\mu \nu} d x^{\mu} d x^{\nu}=-e^{2 u}(d t+a d \varphi)^{2}+e^{-2 u}\left[w^{2} d \varphi^{2}+e^{2 k}\left(d \rho^{2}+d z^{2}\right)\right] .
$$

However, another form, patronized in particular by Bardeen [17], is superior for our purposes, and leads to more similarities with the Newtonian analysis of the same systems. (See the comments about metric (2), later in this section.)

$$
d s^{2}=-e^{2 U} d t^{2}+e^{-2 U}\left[W^{2}(d \varphi-A d t)^{2}+e^{2 K}\left(d \rho^{2}+d z^{2}\right)\right]
$$

with the invariant potentials $U, A, W$, and the conformal factor $e^{2 K}$, all being functions of $(\rho, z)$. (For convective motion a more general metric has to be used, and much less is known about these systems [18]. Even in Newtonian theory such more general dust stars obviously have not been considered.) For the metric (3), one combination of Einstein's field equations reads $W,_{\rho \rho}+W,_{z z}=: \Delta_{2} W=16 \pi e^{2(K-U)} W p$. (In the sequel we use also the flat Laplacians in higher dimensions $n>2: \Delta_{n} f:=$ $\left.f_{, \rho \rho}+f,_{z z}+((n-2) / \rho) f, \rho.\right)$ For dust with $p \equiv 0$ we therefore have $\Delta_{2} W=0$, with the consequence that we can use globally (both in the exterior vacuum region, and in the interior matter region) Weyl coordinates with $W \equiv \rho$. (The fact that this is not possible for pressure-stars, represents another decisive difference between the two cases.) We use a gauge in the $(\rho, z)$-plane such that the rotation axis is given by $\rho=0$. The essential (elliptic) field equations for the potentials $U$ and $A$ read: 


$$
\begin{array}{r}
\Delta_{3} U-\frac{1}{2} \rho^{2} e^{-4 U}|D A|^{2}=4 \pi \epsilon e^{2(K+U)} N^{-1}\left(1+\rho^{2} e^{-4 U} \tilde{A}^{2}\right) \\
\Delta_{5} A-4<D U, D A>=-16 \pi \epsilon e^{2(K+U)} N^{-1} \tilde{A}
\end{array}
$$

with $\tilde{A}=\omega-A, N=e^{4 U}-\rho^{2} \tilde{A}^{2}$, and where $<D U, D A>=U_{, \rho} A,,_{\rho}+U_{,_{z}} A,_{z}$ denotes the Euclidean scalar product of the gradients. Since the time-component of the dust four-velocity is given by $u^{0}=e^{U} N^{-1 / 2}$, we have to have $N>0$, and therefore $\rho|\tilde{A}|<e^{2 U}$, what limits the lateral extension of the dust star. (The velocity of no dust particle may exceed the light velocity.) The conformal factor $e^{2 K}$ appears in equations (4) and (5) only in combination with the energy density $\epsilon$. According to the field equations

$$
\begin{array}{r}
K,_{\rho}=\rho\left(U_{\rho}^{2}-U_{, z}^{2}\right)-\frac{\rho^{3}}{4} e^{-4 U}\left(A,_{\rho}^{2}-A,_{z}^{2}\right) ; \\
K,_{z}=2 \rho U,_{\rho} U,_{z}-\frac{\rho^{3}}{2} e^{-4 U} A,_{\rho} A,_{z},
\end{array}
$$

the potential $K$ results from line integrals as soon as the potentials $U$ and $A$ are known. In the interior matter region $(\epsilon>0)$, the field equations $(4)$ and $(5)$ have to be supplemented by the relativistic Euler equation $\nabla_{\mu} T^{\mu \nu}=0$, leading to the two equations (for $\nu=\rho, z)$

$$
\begin{array}{r}
\left(e^{4 U}+\rho^{2} \tilde{A}^{2}\right) U,_{\rho}=-\rho^{2} \tilde{A}\left(A,,_{\rho}-\tilde{A} / \rho\right) \\
\left(e^{4 U}+\rho^{2} \tilde{A}^{2}\right) U,_{z}=-\rho^{2} \tilde{A} A,_{z} .
\end{array}
$$

(The same equations result from the demand that the geodesic acceleration of the dust particles must not have $\rho$ - and $z$-components.) Equations (8) and (9) comprise an interesting analogy between Einsteinian rotating dust stars and Newtonian rotating stars with pressure: If one divides equations (8) and (9) by $\left(e^{4 U}+\rho^{2} \tilde{A}^{2}\right)$, and compares them with the nonrelativistic Euler equations $U_{, \mu}=-p,_{\mu} / \epsilon+\delta_{\mu \rho} \omega^{2} \rho$ for $\mu=\rho, z$, it is seen that the gravitomagnetic potential $A$ acts like a positive pressure. Differentiating equation (8) with respect to $\rho$, we get a relation between $U, \rho \rho$ and $A, \rho \rho$, and equally a relation between $U_{, z z}$ and $A, z z$ from equation (9). Insertion of these relations into equations (4) and (5) leads to an expression for the energy density, containing only the first derivatives of $U$ and $\omega$ :

$$
4 \pi \epsilon e^{2(K+U)}=\frac{N^{2}}{2 \rho^{2} \tilde{A}^{2}}|D U|^{2}+\frac{N U, \rho}{\rho}+\frac{1}{2} \tilde{A}^{2}+\frac{e^{4 U}}{\tilde{A}}<D \omega, D U>+\frac{\rho \tilde{A} e^{4 U}}{N} \omega,_{\rho} .
$$

Obviously, the last two terms vanish at the axis. 
In the special case of rigid rotation ( $\omega=$ const.) equations (8) and (9) can be explicitly integrated with the result

$$
e^{-2 U} N=e^{2 U_{0}}
$$

where $U_{0}$ denotes the constant potential along the whole interior axis. This constant $U_{0}$ is in principle observable through the redshift $Z_{0}=e^{-U_{0}}-1$ of photons emanating, e.g., from the poles of the star to infinity. (This implies $U_{0}<0$ for a "real" dust star with mass $M>0$.) Equation (11) can be used to express the potential $\tilde{A}$ through the potential $U$ :

$$
\rho \tilde{A}=e^{2 U} \sqrt{1-e^{2\left(U_{0}-U\right)}} .
$$

Insertion of equation (12) into the field equations (4) and (5) even results (only in the matter region!) in an elliptic equation solely for $U$, where also $K$ and $\epsilon$ cancel out. For rigid rotation the above expression (10) for $\epsilon$ simplifies to

$$
\epsilon=\frac{1}{8 \pi \rho^{2}} e^{4 U_{0}+2 U-2 K}|D \tilde{B}|^{2}
$$

with $\tilde{B}=\rho e^{-2 U_{0}} \sqrt{1-e^{2\left(U_{0}-U\right)}}$. In this case, $\epsilon$ therefore is automatically nonnegative. Since the potentials $U$ and $A$ have to be continuously differentiable at the surface of the (hypothetical) dust star (no mass or angular momentum shells!), equation (12) together with its derivative normal to the star's surface could be used as boundary conditions for the solution of the exterior vacuum equations (equations (4) and (5) with $\epsilon=0$ ). Since, however, the boundary condition (12) for dust stars differs only slightly from the corresponding boundary condition for stars with small pressure (for which we expect a transition from nonexistence to existence of physical solutions $U$ and $A$ ), we doubt the usefulness of these boundary conditions, even in the case of rigid rotation.

Instead, we see from equation (9) that also for differential rotation the term $U_{, z}$ is zero along the interior axis $(\rho=0)$, and therefore $U \equiv U_{0}$ at this axis. Although, according to equation (8), also $U, \rho$ is zero at the interior axis (no "gravitational force" there!), the ratio $U_{, z} / U_{, \rho}=\rho A_{, z} /\left(\rho A,_{\rho}+A-\omega\right)$ is zero there if $|\omega|>|A|$, as is to be expected on physical grounds (compare item e) below). Therefore, the decisive (geometric) difference between dust stars and pressure stars shows up in the structure of the level lines of the potential $U$ : In the dust region they are parallel to the rotation axis in its neighborhood, whereas they are orthogonal to the axis in the exterior, resulting in a type of singularity of the level lines at the poles. In contrast, for pressure stars the $U$ level lines are everywhere orthogonal to the axis, without any singularity at the poles.

For the metric form (2) the potential $u$ in vacuum obeys the differential equation 
$\Delta_{2} u=-e^{4 u}|D a|^{2} / 2 \rho^{2}$, being singular at the axis, so that not all solutions can be analytic; the potential $a$ has, with $r=\sqrt{\rho^{2}+z^{2}}$, and with the angular momentum $J$, the asymptotic behavior $a \longrightarrow 2 J \rho^{2} / r^{3}$, being different for axis- and off axisdirections, what does not happen for the potential $A \longrightarrow 2 J / r^{3}$; and - most important - the potential $u$ is not constant along the interior axis, so that the analysis of the $u$ level lines does not lead to comparably useful results.

Before we take (in Secs. 3 - 4) first steps to a nonexistence proof for rotating dust stars, we should like to summarize here some mathematical properties of the potentials $U$ and $A$ in the exterior and interior of the hypothetical dust star.

a) Due to the axial symmetry, the potentials depend only on $\rho^{2}$.

b) For a real rotating star, with mass $M>0$, and angular momentum $J \neq 0$, the potentials have the asymptotic behavior $U \longrightarrow-M / r, A \longrightarrow 2 J / r^{3}$, with $r=\sqrt{\rho^{2}+z^{2}}$. According to equation (4) with $\epsilon=0$, the rotation, i.e. the term $|D A|^{2}$, contributes to the asymptotic behavior of $U$ only in order $r^{-4}$.

c) In the vacuum region, equations (4) and (5) do not explicitly depend on $z$, and in the matter region an explicit $z$-dependence can only come from the angular velocity $\omega\left(\rho^{2}, z\right)$. Of course the solutions $U$ and $A$ will depend on $z$, e.g., in order to fulfil the asymptotic conditions. However, at least in the exterior, for any solution $\left(U\left(\rho^{2}, z\right), A\left(\rho^{2}, z\right)\right)$ there exists also a solution $\left(U\left(\rho^{2}, z-z_{1}\right), A\left(\rho^{2}, z-z_{1}\right)\right)$ with an arbitrary constant $z_{1}$. And we can fix the origin of our coordinate system $(\rho, z)$ such that it lies in the matter region.

d) According to a mathematical theorem by Morrey [19], made applicable to general relativity by Müller zum Hagen [20], $U$ and $A$ are real analytic functions in the exterior. Therefore $U$ and $A$ in the exterior are determined by their axis values, as can be explicitly confirmed by writing them as (convergent!) power series' in, e.g., $\tau=1-z / r$. Whereas for stars with pressure the differentiability class of $U$ and $A$ in the matter region is connected with the differentiability of the equation of state $\epsilon(p)$ (according to Proposition 5 of [21], $U$ and $A$ are of the class $\mathbb{C}^{k+2}$ if $\epsilon(p)$ is of class $\left.\mathbb{C}^{k}\right)$, for dust stars also the interior potentials $U$ and $A$ are real analytic, if the angular velocity $\omega\left(\rho^{2}, z\right)$ is an analytic function, what we like to presume. Evolving equations (4)-(5) and (8)-(9) in powers of $s=\rho^{2}$, one finds that in the expression $\omega(s, z)=\omega_{0}+\omega_{1}(z) s+\omega_{2}(z) s^{2}+\ldots$ the first term $\omega_{0}$ is constant, and each $\omega_{n}(z)$ produces one new integration constant $c_{n}$. The functions $U, A, \epsilon$, and $\omega_{n}(z)$ for $n>1$ can then be expressed in the whole dust region through the function $\omega_{1}(z)$ and its derivatives, together with the constants $c_{n}$. (In the case $\omega_{1}(z) \equiv 0$, the function $\omega_{2}(z)$ takes over the role of this central free function. In the case $\omega=$ const., i.e. $\omega_{1}=\omega_{2}=\ldots \equiv 0$, the energy density $\epsilon_{0}(z)$ at the axis takes over this 
role, as was already stated by Gürlebeck [12].) Across the surface, $U$ and $A$ are only continuously differentiable, at least if $\epsilon>0$ at the surface.

e) According to the weak maximum principle (see, e.g., [22], Theorem 3.1), equations (4) and (5) tell that there can be no maxima of $U$, and no maxima or minima of $A$ within the exterior vacuum region. Whether $U$ can have minima there, is "the big question" which was already mentioned in the Introduction, and to which we will come back in Sec. 4. It is clear that minima of second order cannot appear on the axis because there we have $\Delta_{3} U=0$, and therefore different signs of $U_{, \rho \rho}$ and $U_{, z z}$. Since the rhs of equation (4) is positive in the interior dust region, $U$ can also not have maxima there. Equation $(7)$ together with $K \longrightarrow 0$ at infinity leads to $K(0, z)=0$ on the whole axis. Equation (4) then yields $U(\rho, z)=U_{0}+\pi e^{-2 U_{0}} \epsilon(0, z) \rho^{2}+O\left(\rho^{4}\right)$ near the interior axis, so that $U$ increases there for a dust star with $\epsilon(0, z)>0$. (Insertion of the above $U(\rho, z)$ near the axis into equation (10) leads to $2 \pi \epsilon(0, z)=\tilde{A}^{2}(0, z) e^{-2 U_{0}}+O\left(\rho^{2}\right)$.) Together with the absence of maxima, we get $U>U_{0}$ on the whole star interior and surface outside the axis. If we apply the weak maximum principle for generalized solutions (see, e.g., [22], Theorem 8.1) to equation (4) for a ball with very large radius around the origin, we get for a bounded $U$ (no singularities!) with $U \longrightarrow 0$ at infinity: $-\infty<U \leq 0$. In the case of rigid rotation we can substitute $-\tilde{A}$ for $A$ in the lhs of equation (5). Again applying the weak maximum principle to this equation, and using $A \longrightarrow 0$ at infinity, we get (for $\omega>0$ ) : $0 \leq A \leq \omega$. (Dragging velocity smaller than or equal to the dust velocity.) For differential rotation we can expect similar inequalities at most in some averaged sense because we can then choose $\omega$ arbitrarily small, or even negative, in some local region.

Before we perform (in Sec. 3) an analysis of the potential $U$ near the pole of a rotating dust star, and address (in Sec. 4) the question of minima of $U$ in the exterior vacuum region, we like to present here a simple nonexistence proof for static dust stars in general relativity. In the static case $(\omega=0)$ there exists of course also no gravitomagnetic potential: $A \equiv 0$. Then equations (8) and (9) tell that $U$ is constant in the dust region. Herewith we have $\Delta_{3} U=0$, and from equation (4) $\epsilon \equiv 0$, i.e., no real dust star. (The analysis in the matter region suffices here, as was the case for the nonexistence proofs by Gürlebeck [12].) An alternative nonexistence proof for static dust stars (using the positive energy theorem) was given by Shiromizu [23], and in [24] a concrete threshold for $p / \epsilon$ (in dependence of $U$ ) was given maintaining nonexistence. 


\section{Analysis of the potential $U$ near the pole of the dust star}

As already indicated in Secs. 1 and 2, a decisive means on the way to a nonexistence proof for rotating dust stars should be the study of the level lines of the $U$-potential near the north pole $\left(\rho=0, z=z_{0}\right)$. Up to now we are successful in this analysis only if we put some (mild) restrictions on the model class of the rotating dust stars: a) We require that the star surface near the north pole allows for an osculating sphere, i.e., that we have no cone or cusp there. b) The energy density $\epsilon(0, z)$ at the axis should either be positive at the pole $z_{0}$ (hard surface), or one of its $z$-derivatives should be non-zero at $z_{0}$. (Otherwise the energy density $\epsilon(0, z)$ near $z_{0}$ had to have a very unusual behavior like $e^{1 /\left(z-z_{0}\right)}$.) According to item d) of Sec. 2 the basic degrees of freedom of the rotating dust are given by a series of constants $c_{n}$, by the constant $\omega_{0}$, and by the function $\omega_{1}(z)$ in a power series $\omega\left(\rho^{2}, z\right)=\omega_{0}+\omega_{1}(z) \rho^{2}+\omega_{2}(z) \rho^{4}+\ldots$. Due to $\epsilon(0, z)=\tilde{c} \omega_{1}^{2}(z)$, with a positive constant $\tilde{c}$, the above conditions on $\epsilon(0, z)$ are lastly conditions on the function $\omega_{1}(z)$ at $z=z_{0}$ (or on $\omega_{2}(z)$, in the case $\omega_{1} \equiv 0$ ).

We consider now equation (4) in the dust and in the vacuum region near the axis $\rho=0$. There we have $K \approx 0, N \approx e^{4 U}, U\left(\rho^{2}, z\right)-U(0, z) \approx \rho^{2} f(0, z)$, and therefore $\Delta_{3} U \approx 2 U,_{\rho \rho}+U, z z$, where the symbol $\approx$ indicates that in the limit $\rho \longrightarrow 0$ any correction terms vanish more strongly (typically an order $\rho^{2}$ stronger) than the terms explicitly denoted. (The validity of these approximations may be interpreted as another — mild - restriction for our class of rotating dust stars.) Then equation (4) reduces near the axis to

$$
2 U_{, \rho \rho}+U_{, z z} \approx 4 \pi e^{-2 U_{0}} \epsilon(0, z) .
$$

In the dust region we have, due to $U(0, z) \equiv U_{0}$ the result $U, z z \equiv 0$, and therefore $U_{, \rho \rho} \approx 2 \pi e^{-2 U_{0}} \epsilon(0, z)$. For a surface with sphere condition at the north pole $U,{ }_{\rho \rho}$ is continuous across this surface. Therefore we have in the vacuum region near the north pole $z=z_{0}$ :

$$
U,_{z z} \approx-2 U,_{\rho \rho} \approx-4 \pi e^{-2 U_{0}} \epsilon\left(0, z_{0}\right) .
$$

In the case $\epsilon\left(0, z_{0}\right)>0$ we have $U_{, z z}\left(0, z_{0}\right)<0$, so that $U\left(0, z>z_{0}\right)$ falls below the value $U_{0}<0$. Together with $U \longrightarrow 0$ at infinity, and with the analyticity of $U\left(\rho^{2}, z\right)$ in the vacuum region, this enforces a minimum of $U\left(\rho^{2}, z\right)$ there. Since near the north pole $U\left(\rho^{2}, z\right)$ has its steepest descent in the positive $z$-direction, one may argue also on the basis of symmetry reasons - that this $U$-minimum should be reached at an axis-point. But on the axis we have $U_{, z z}=-2 U_{, \rho \rho}$, what forbids minima of the second order. Therefore solutions of this "most simple type" can already be excluded, i.e., nontrivial rotating dust star solutions of this type cannot exist. More generally, from equation (15) in the case $\epsilon\left(0, z_{0}\right)>0$ follows that the vacuum solution $U\left(\rho^{2}, z\right)$ near the north pole is given by $U\left(\rho^{2}, z\right) \approx U_{0}+\pi e^{-2 U_{0}} \epsilon\left(0, z_{0}\right)\left(\rho^{2}-2 z^{\prime 2}\right)$, 
with $z^{\prime}=z-z_{0}$. Therefore the level line $U=U_{0}$, which is the $z$-axis in the dust, bifurcates at the north pole $z=z_{0}$ into the two branches $z^{\prime} \approx \pm \rho / \sqrt{2}$. What can be said about the continuation of this bifurcating level line $U=U_{0}<0$ into the vacuum region? i) This level line cannot terminate inside the vacuum region because, as an analytic curve, it would allow an analytic continuation beyond such an "end-point". ii) It cannot extend to infinity because there we have $U \longrightarrow 0$. iii) It cannot "return" to the star surface because there we have $U>U_{0}$ outside the axis. Therefore we are left with two other realistic possibilities: iv) The level line closes within the vacuum region without enclosing the dust region. Then it encloses a finite vacuum domain with $U<U_{0}$, in which there exists (due to the analyticity of $U$ ) a $U$-minimum. v) The level line $U=U_{0}$ encloses the whole dust region, and has $U<U_{0}<0$ in its exterior. Due to $U \longrightarrow 0$ at infinity, there has then to exist a second closed level line $U=U_{0}$ exterior to the first one. And between these two level lines there has again to exist a minimum of $U$.

For the stabilized Newtonian dust star we have shown in [1] that it realizes the alternative iv): The level line $U=U_{0}$ starts as the $z$-axis in the dust region, and bifurcates at the pole $\left(\rho=0, z=R_{1}\right)$ into two branches with slope $z^{\prime}= \pm \rho / \sqrt{2}$ (because $\epsilon>0$ at the dust surface for this model). The outer ring of strained matter in the region $R_{2} \leq r \leq R_{3}$ "bends back" these branches to one closed curve which includes a region with $U<U_{0}$, containing a $U$-minimum at $\left(\rho=0, z=R_{2}\right.$ ). (See Fig. 3 of [1]. This $U$-minimum at the axis is not in contradiction to the statement under e) of Sec. 2, excluding such minima in the vacuum region, because now we have matter in the region $R_{2} \leq r \leq R_{3}$, leading to a discontinuity of $\Delta_{3} U$ at $r=R_{2}$.)

In the case $\epsilon\left(0, z_{0}\right)=0$, but $-4 \pi e^{-2 U_{0}} \partial^{j} \epsilon(0, z) / \partial z^{j}=: \tilde{\epsilon}>0$ at $z_{0}$ for some integer $j \geq 1$, the potential $U\left(\rho^{2}, z\right)$ is $(j+1)$ times continuously differentiable with respect to $z$ near the pole, and the $(j+2)^{t h}$ derivative has a finite discontinuity there. Then we can take the $j^{\text {th }}$ z-derivative of equation (14), and get (like above for the case $\left.\epsilon\left(0, z_{0}\right)>0\right)$ in the vacuum region near the north pole, with $V\left(\rho^{2}, z\right):=$ $\partial^{j} U\left(\rho^{2}, z\right) / \partial z^{j}: V_{, z z} \approx-2 V, \rho \rho \approx \tilde{\epsilon}$. Therefore $U\left(\rho^{2}, z\right)$ is given there by $U\left(\rho^{2}, z\right) \approx$ $U_{0}-\tilde{\epsilon} z^{\prime j}\left[\rho^{2} / 4 j !-z^{\prime 2} /(j+2) !\right]$, and the level line $U=U_{0}$, being the $z$-axis in the dust, divides at the north pole into the 3 branches $z^{\prime} \approx 0$, and $z^{\prime} \approx \pm \frac{1}{2} \sqrt{(j+1)(j+2)} \rho$. In the area $0<\left|z^{\prime}\right|<\frac{1}{2} \sqrt{(j+1)(j+2)} \rho$ near the north pole the potential $U\left(\rho^{2}, z\right)$ falls below the value $U_{0}<0$, and therefore has to attain a minimum somewhere in the exterior vacuum region.

Herewith we have shown that, at least for the restricted class of rotating dust stars as characterized at the beginning of this section, the existence of such stars 
(with $M>0$ ) in general relativity leads to a minimum of the potential $U\left(\rho^{2}, z\right)$ in the exterior vacuum region.

\section{The question of minima of the potential $U$ in the exterior vacuum region}

In this section we analyze the Einstein equations for stationary, axisymmetric, and asymptotically flat systems only in the vacuum region where equations (4) and (5) simplify to

$$
\begin{aligned}
& \Delta_{3} U=\frac{1}{2} \rho^{2} e^{-4 U}|D A|^{2} ; \\
& \Delta_{5} A=4<D U, D A>.
\end{aligned}
$$

And the central question concerns possible minima of the (quasi-Newtonian) potential $U$, because the exclusion of such minima would, according to Sec. 3, and by the principle of contradiction, imply that rotating dust stars (at least of the mildly restricted class considered here) do not exist in general relativity. From a qualitative physical point of view, a minimum of $U$ in the exterior vacuum region would be hardly imaginable anyhow: Which place $(\bar{\rho}, \bar{z})$ in the largely "structureless" vacuum region should be distinguished by a maximal gravitational attraction in the direction of the dust part? However, we are of course looking for a strict mathematical non-existence proof for $U$-minima.

Although the stationary and axisymmetric vacuum Einstein equations are presumably the systems in general relativity which have been studied most thoroughly over many years, and about which there exists a huge literature (see, e.g., [16], and literature quoted there), obviously no mathematical proof is available hitherto excluding minima of the potential $U$ in the general case. (It is, however, easy to prove that for the Schwarzschild and Kerr geometries, written in our metric form (3), the potential $U$ has no minima.) The mathematical literature (at least in the form of textbooks and review articles) on coupled, nonlinear, and elliptic systems of differential equations seems, anyhow, to be quite sparse. The textbook [25] treats (in Chap. 8) such systems, which are even applicable to the Einstein equations, since they are typically bilinear in the first derivatives of the "potentials". However, the question of extrema of the solutions is not addressed there. Moreover, the Einstein equations have a very special structure (with hidden symmetries) so that general mathematical theorems about differential equations are usually of little profit. Admittedly, the transcription of the stationary and axisymmetric vacuum Einstein equations to the Ernst equation, and its treatment with inverse and algebro-geometric methods (see, e.g., [26]) takes advantage of this special structure. Since, however, these methods 
are intrinsically local, they obviously give no answer to our global problem concerning minima of $U$. We have tested local expansions of the potentials $U$ and $A$ around the north pole, around the hypothetical minimum position of $U$, and around infinity. But in each case there remain too many "degrees of freedom", so that solutions of the system (16)-(17) with a $U$-minimum cannot be excluded in this way. Also the fact that the system (16)-(17) does not explicitly depend on $z$, what produces for any solution $\left(U\left(\rho^{2}, z\right), A\left(\rho^{2}, z\right)\right)$ corresponding solutions $\left(U\left(\rho^{2}, z-z_{1}\right), A\left(\rho^{2}, z-z_{1}\right)\right)$, with arbitrary constants $z_{1}$, seems not to be helpful for our goal.

Therefore, we see two remaining strategies for excluding $U$-minima:

i) One can directly study the mathematical structure of the system of differential equations (16)-(17). The comparison with the stabilized Newtonian dust star in [1] shows that in equation (16) the term $|D A|^{2}$ (with the asymptotic behavior $r^{-8}$ ) acts like a positive mass density which falls off in all asymptotic directions. Since the analysis in [1] has shown that there exist exterior mass distributions which stabilize the (Newtonian) rotating dust star, and lead to $U$-minima in this region, an exclusion of $U$-minima can only result from appropriate restrictions of the gradient of the potential $A$ in equation (16), and such restrictions obviously can only come from equation (17). The sought after restriction on $A$ is, however, not of the type that the absolute value of $A$ or $|D A|$ is restricted, because the factor $\frac{1}{2}$ in equation (16), or any other positive constant $C^{2}$ could be integrated into a new potential $A^{\prime}\left(\rho^{2}, z\right)=C A\left(\rho^{2}, z\right)$, which again fulfils the homogeneous equation (17). And on physical grounds we like to consider (and exclude) dust stars with nearly arbitrary angular velocity $\omega$. Therefore, no a priori restriction on the values of $A\left(\rho^{2}, z\right)$ seems possible, besides the condition that the velocity of the equatorial dust particles must not exceed the light velocity $c$, what is not a stringent restriction, at least for "small stars". It should also be mentioned that the insertion of dimensional quantities into equation (16) changes $A$ to $A / c$, what also explains why the Newtonian nonexistence problem is so much simpler than the Einsteinian problem. (Compare the Newtonian nonexistence proofs in the Introduction.)

ii) A second strategy for excluding $U$-minima may be the transcription of the equations (16)-(17) to a "simpler system". The substitution $S=e^{2 U}$ leads to the system

$$
\begin{gathered}
S \Delta_{3} S=|D S|^{2}+\rho^{2}|D A|^{2} ; \\
S \Delta_{5} A=2<D S, D A>,
\end{gathered}
$$

what consititutes presumably the structurely simplest form of our problem, equation (18) being purely quadratic in $S$ and $A$, and equation (19) being homogeneously linear in both potentials. By the substitution $B=\rho A$ we can produce flat 3dimensional Laplacians in both equations, but only at the cost of additional terms with $\rho$-derivatives. We mentioned already that the transcription of the system (16)- 
(17) to the Ernst equation promises no progress in the exclusion of $U$-minima. In analogy to work by Weinstein [27] one may think of an application of harmonic mappings to these equations. However, direct application of the formalism of [27] does not seem to be successful because results about extrema of the potential $X=\rho^{2} e^{-2 U}$, appearing there, say nothing about $U$-minima. A very ambitious goal (which was successfully achieved in some mathematical problems of similar structure) is a "decoupling" of equations (16) and (17), i.e., the discovery of a separate differential equation for some (obviously nonlinear) combination of $U$ and $A$, and of their gradients. For instance, one can think of energy-like expressions as they appear in the components of the Landau-Lifshitz pseudotensor for the gravitational field. However, hitherto we did not yet succeed with this attempt. (The transcription to the Ernst equation leads only seemingly to such a decoupling because it contains besides the complex Ernst potential $\mathcal{E}$ also the real part $\operatorname{Re}(\mathcal{E})=e^{2 u}$.) Should some decoupling turn out to be realizable finally, it would presumably also lead to a better understanding and a further simplification of the algebro-geometric methods as applied to the Ernst equation.

\section{Acknowledgements}

I am deeply indebted to Urs Schaudt who some years ago provided essential ideas to this paper, in particular the proposal to consider the level lines of the $U$-potential. In more recent times I profited from discussions with P. Chrusciel, J. Frauendiener, N. Gürlebeck, G. Huisken, C. Klein, O. Reula, and B. Schmidt.

\section{References}

[1] Schaudt U M and Pfister H 2001 Gen. Rel. Grav. 33719

[2] Bonnor W B 1977 J. Phys. A 101673

[3] Pfister H and Schedel C 1987 Class. Quantum Grav. 4141

[4] Ehlers J 1991 in: Classical Mechanics and Relativity: Relationship and Consistency ed G Fenarese (Naples: Bibliopolis) pp 95 - 106

[5] Caporali A 1978 Phys. Letters A 665

[6] Binney J and Tremaine S 1994 Galactic Dynamics (Princeton: Princeton University Press) Chap. 5.3 
[7] Neugebauer G and Meinel R 1993 Astrophys. J. 414 L97

[8] Lanczos K 1924 Zeitschr. f. Physik 21 73; English translation in Gen. Rel. Grav. 29 (1997) 363

[9] van Stockum W J 1937 Proc. Roy. Soc. Edinb. 57135

[10] Winicour J 1975 J. Math. Phys. 161806

[11] Frauendiener J 1987 Phys. Letters A 120119

[12] Gürlebeck N 2009 Gen. Rel. Grav. 412687

[13] Heilig U 1995 Comm. Math. Phys. 166457

[14] Pfister H and Schaudt U M 2000 Ann. Phys. (Leipzig) 11507

[15] Kundt W and Trümper M 1966 Zeitschr. f. Physik 192419

[16] Stephani H et al. 2003 Exact Solutions of Einstein's Field Equations (Cambridge: Cambridge University Press)

[17] Bardeen J 1970 Astrophys J 16271

[18] Gourgoulhon E and Bonazzola S 1993 Phys. Rev. D 482635

[19] Morrey C B 1958 Am. J. Math. 80198

[20] Müller zum Hagen H 1970 Proc. Camb. Phil. Soc. 68199

[21] Schaudt U M 1998 Comm. Math. Phys. 190509

[22] Gilbarg D and Trudinger N S 1983 Elliptic Partial Differential Equations of Second Order, Second Edition (Berlin: Springer)

[23] Shiromizu T 1994 Class. Quantum Grav. 11 L29

[24] Shiromizu T and Yoshino H 2007 Progr. Theor. Phys. 1161159

[25] Ladyzhenskaya O A and Uraltseva N N 1968 Linear and Quasilinear Elliptic Equations (New York: Academic Press)

[26] Klein C and Richter O 2005 Ernst Equation and Riemann Surfaces (Berlin: Springer)

[27] Weinstein G 1990 Comm. Pure Appl. Math. 43903 